\title{
Pathogenic synergy between Escherichia coli and Bacteroides fragilis or $B$. vulgatus in experimental infections: a non-specific phenomenon
}

\author{
A. M. J. J. VERWEIJ-VAN VUGHT, F. NAMAVAR, W. A. C. VEL, M. SPARRIUS and D. M. \\ MACLAREN
}

Research Group for Commensal Infections, Departments of Medical and Oral Microbiology, Schools of Medicine and Dentistry, Vrije Universiteit, P.O.Box 7161, 1007 MC Amsterdam, The Netherlands

\begin{abstract}
Summary. The virulence of Bacteroides fragilis and B. vulgatus for mice was compared in a skin-infection model. These strains were also tested for pathogenic synergy in mixed infections with Escherichia coli. Strains of $B$. fragilis were generally more virulent than strains of $B$. vulgatus and, with one exception, the effect of Bacteroides strains in mixed infections merely reflected their inherent virulence.
\end{abstract}

\section{Introduction}

The normal microbial intestinal flora determines the initial inoculum in intra-abdominal infections from which with careful laboratory handling an average of four to five species may be isolated (Finegold, 1977). The pathogenic significance of each isolate remains for the most part unclear.

The frequent presence of Escherichia coli and Bacteroides species of the fragilis group in these infections has led to the concept of pathogenic synergy between them (Gorbach and Bartlett, 1974b; MacLaren et al., 1984). With various experimental animal models, evidence has been presented to support this theory (Onderdonk et al., 1976; Kelly, 1978; Verweij-van Vught et al., 1985). No satisfactory explanation has been given for the higher incidence of $B$. fragilis in mixed intraabdominal infections compared with other species of the fragilis group, i.e., B. vulgatus, B. distasonis, $B$. ovatus and $B$. thetaiotaomicron, despite the lower prevalence of $B$. fragilis in the intestinal flora (Gorbach and Bartlett, 1974a).

The higher virulence of $B$. fragilis as documented by Onderdonk et al. (1977) and Maskell (1981) may explain the higher incidence of this species in infections. Evidence has been given for an important role of the capsular polysaccharide of $B$. fragilis as a virulence factor (Onderdonk et al., 1977; Brook and Walker, 1984). On the other hand, the possibility remains of a synergistic mechanism specific for $B$. fragilis. In this paper, differences in pathogenic synergy between $E$. coli and $B$. fragilis and between $E$. coli and $B$. vulgatus are described. These differ-

Received 18 Feb. 1985; accepted 28 Mar. 1985. ences have been studied in relation to the virulence of strains of Bacteroides in infections caused by a single species.

\section{Materials and methods}

\section{Bacterial strains}

The Bacteroides strains used in this study were collected from intra-abdominal wound infections of patients at the Academic Hospital of the Vrije Universiteit (BE strains 1, $12,13,21,43$ and 53) or from faeces from the same patient population (BE strains $16,17,18,19$ and 20). E. coli strain EB 1 was isolated together with $B$. fragilis strain BE 1 from an intra-abdominal wound infection. Staphylococcus aureus strain BE 50 was also isolated from a wound infection. The $E$. coli strain was identified with the API 20 E system (API S.A., Montalieu Vercieu, France) and the Bacteroides strains with the BBL Minitek ${ }^{\circledR}$ Numerical Identification system (BBL Microbiology Systems, Becton Dickinson, USA).

\section{Animals}

Swiss Albino mice (TNO, Zeist, The Netherlands) weighing $20-25 \mathrm{~g}$ were used. The mice were raised in conventional conditions.

\section{Inoculum preparation}

$E$. coli strain EB 1 and $S$. aureus strain BE 50 were cultured aerobically in Nutrient Broth (No. 2, Oxoid) until in the late logarithmic phase of growth. Cells were harvested by centrifugation, washed in phosphate-buf-

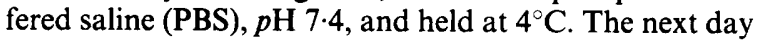
an appropriate dilution, based on the viable count of the suspension, was made in PBS. B. fragilis and B. vulgatus 
were grown in BM medium (Shah et al., 1976) supplemented with haemin $5 \mathrm{mg} / \mathrm{L}$ and menadione $2 \mathrm{mg} / \mathrm{L}$ at $37^{\circ} \mathrm{C}$ in an anaerobic jar with an atmosphere of $\mathrm{N}_{2} 80 \%$, $\mathrm{H}_{2} 10 \%$ and $\mathrm{CO}_{2} 10 \%$. To prevent excessive acid production, glucose was omitted from the medium and $\mathrm{K}_{2} \mathrm{HPO}_{4}$ was added at a concentration of $5 \mathrm{~g} / \mathrm{L}$. A 20 -h culture, diluted 1 in 20 in fresh medium and incubated for $c .4 \mathrm{~h}$, gave a logarithmic-phase culture. These cells were harvested by centrifugation and resuspended in PBS. A 0.1 $\mathrm{ml}$ volume of appropriate dilution of the bacteria, alone or in combination, was injected subcutaneously in the backs of mice about $1 \mathrm{~cm}$ from the midline after the skin had been shaved.

\section{Evaluation of abscess formation and clearance}

At different times after injection, animals were killed by chloroform inhalation, the skin was cleaned with ethanol $70 \% \mathrm{v} / \mathrm{v}$ and allowed to dry. Skin fragments including the site of the lesion were excised and examined for pus formation. Only pus-containing abscesses were regarded as positive. The fragments were incised through the centre and homogenised (Thomas Tissue Grinder, Philadelphia, USA) in PBS ( $5 \mathrm{ml})$. Viable counts were made: for $E$. coli on nutrient agar incubated aerobically; for Bacteroides spp. on BM medium supplemented with haemin $5 \mathrm{mg} / \mathrm{L}$, menadione $2 \mathrm{mg} / \mathrm{L}$ and nalidixic acid $50 \mathrm{mg} / \mathrm{L}$ incubated anaerobically. In this way, clearance of bacteria was determined.

\section{Results}

In previous studies on the influence of $B$. fragilis on the clearance of $E$. coli from the skin of mice, stationary-phase cultures incubated for $18-20 \mathrm{~h}$ were used. However, comparison of viable and total counts of these cultures revealed that their viability varied between 10 and $100 \%$. Because the presence of a variable and unknown number of dead bacteria may influence the course of infection by Bacteroides spp. and the clearance of $E$. coli in mixed infections, only cells from early logarithmic-phase cultures were used in further experiments. With these cultures, discrepancies between viable and total counts were never observed.

\section{Virulence of B. fragilis and B. vulgatus}

The virulence of strains of Bacteroides was assessed in a mouse model. The decrease in numbers of bacteria in the skin of mice after subcutaneous injection of $c .2 \times 10^{8} \mathrm{cfu}$ was followed for 3 days. For five of six strains of $B$. fragilis, numbers decreased relatively slowly by factors of $10^{1}-10^{3}$ after 3 days (fig. 1). One strain of $B$. fragilis and five strains of $B$. vulgatus were cleared more effectively, their numbers decreasing by factors of $10^{5}-10^{7}$ in the same period (fig. 1).

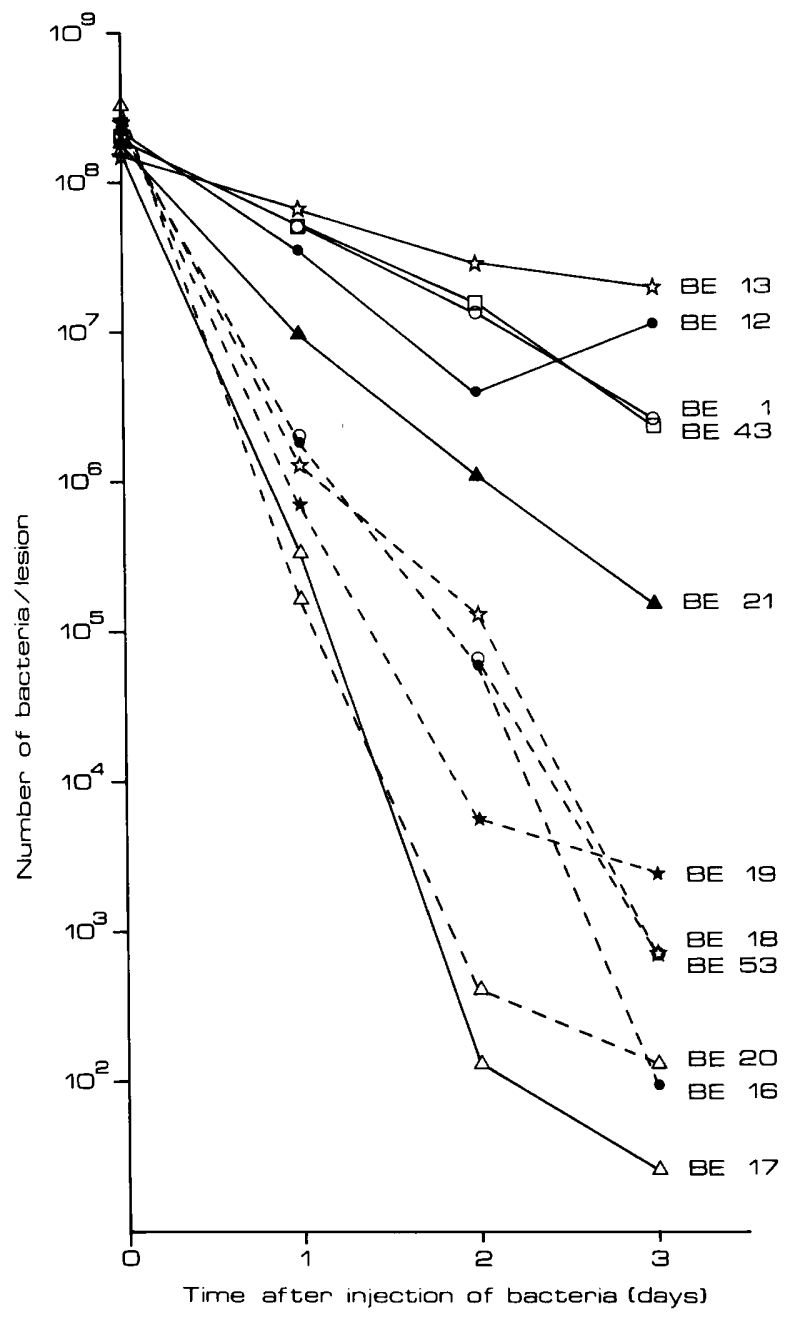

Fig. 1. - The clearance of B. fragilis (-) and B.vulgatus (-- ) from the skin of mice at 1,2 and 3 days after injection of (24) $\times 10^{8} \mathrm{cfu}$. Each point represents the mean of 6-25 lesions.

Attempts to determine LD50 values were less satisfactory, indicating values greater than $10^{9} \mathrm{cfu}$ for B. fragilis strain BE 1 and B. vulgatus strain $\mathrm{BE}$ 18.

\section{Pathogenic synergy between E. coli and Bacteroides species}

After injection of either $E$. coli strain EB 1 (c. $\left.5 \times 10^{6} \mathrm{cfu}\right)$ or B. fragilis strain BE $1\left(\right.$ c. $\left.2 \times 10^{8} \mathrm{cfu}\right)$, each species was cleared effectively from the skin of mice (fig. 2). Their injection together resulted in abscess formation in all mice and after 6 days large

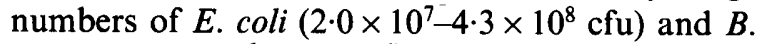
fragilis $\left(3.5 \times 10^{6}-5.5 \times 10^{7} \mathrm{cfu}\right)$ were present in the abscesses. When B. vulgatus strain BE 18 and E. coli strain EB 1 were injected together, their clearance 


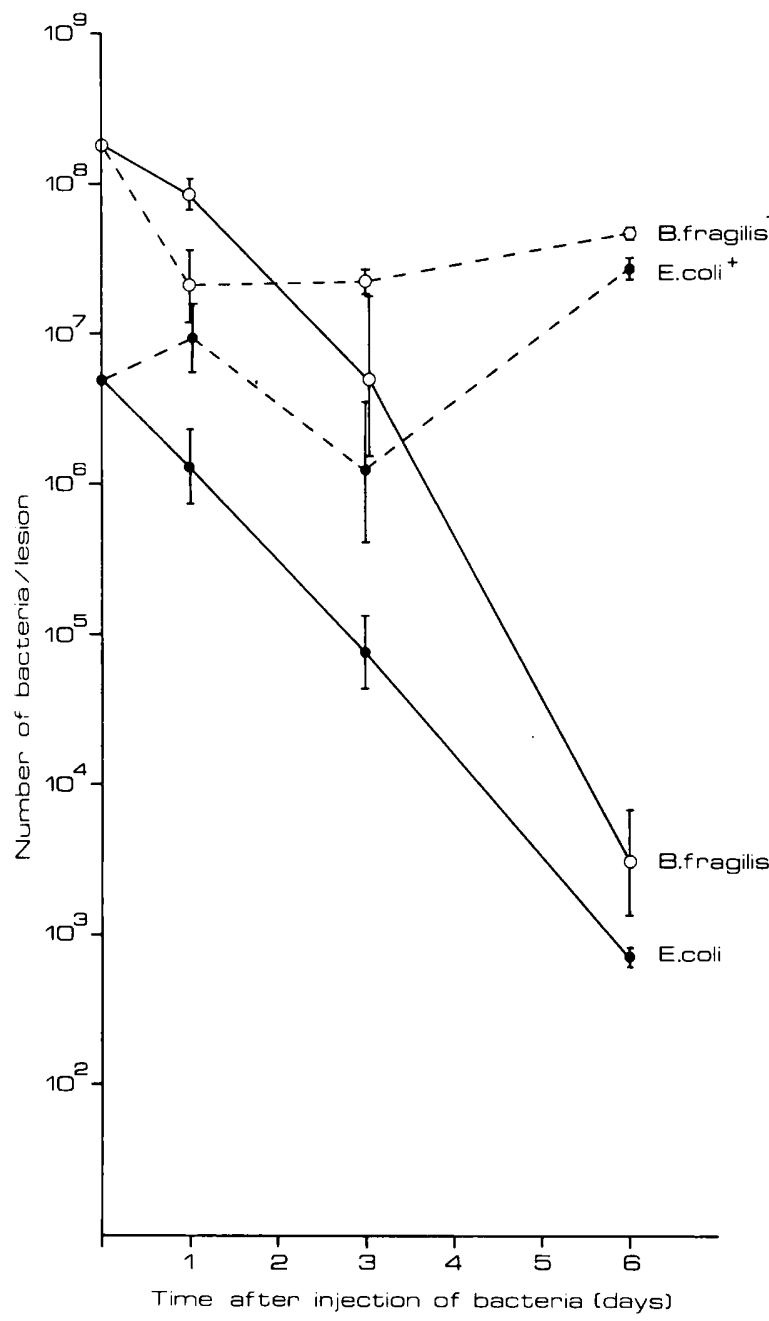

Fig. 2. - The clearance of E. coli strain EB $1(\bullet)$ and B. fragilis strain BE $1(O)$ after injection alone $(-)$ or together $(---)$. Each point represents the mean of at least six lesions and bars indicate the standard error of the mean (SEM).

was initially delayed in all mice (fig. 3). Thereafter, the response varied. Thus, some mice developed abscesses whereas in others lesions tended to heal during the course of the experiment so that, after 6 days, only $50 \%$ of the mice had developed abscesses. The numbers of bacteria in the lesions. varied correspondingly, ranging from $5.0 \times 10^{3}$ to $1.6 \times 10^{7}$ cfu for $E$. coli and from 0 to $2.0 \times 10^{6} \mathrm{cfu}$ for $B$. vulgatus.

Injection of a smaller dose $\left(2 \times 10^{6} \mathrm{cfu}\right)$ of $E$. coli strain EB 1 along with $c .2 \times 10^{8} \mathrm{cfu}$ of $B$. fragilis strain BE 1 or $B$. vulgatus strain BE 18 resulted in the formation of fewer abscesses. Thus, with $B$. fragilis, only $50 \%$ of the mice developed abscesses after 6 days whereas with $B$. vulgatus abscesses were not found at all.
Each of four strains of $B$. fragilis and four strains of B. vulgatus $\left(c .2 \times 10^{8} \mathrm{cfu}\right)$ was tested along with $E$. coli strain EB $1\left(2 \times 10^{6} \mathrm{cfu}\right)$ and the numbers of bacteria in the skin lesions followed for up to 6 days. Control experiments included tests with each strain injected alone. The mean of the numbers of $E$. coli strain EB 1 in the lesions 1, 3 and 6 days after injection is given in fig. 4. Each strain of Bacteroides impaired the clearance of $E$. coli and, except for $B$. fragilis strain BE 17, this influence was significant at a level of $p=0.01$ (as tested by the non-parametric test of Wilcoxon or the U-test of Mann and Whitney). There was a strong correlation between the observed virulence of Bacteroides strains in infections when injected alone and their influence on the clearance of $E$. coli; this effect was most

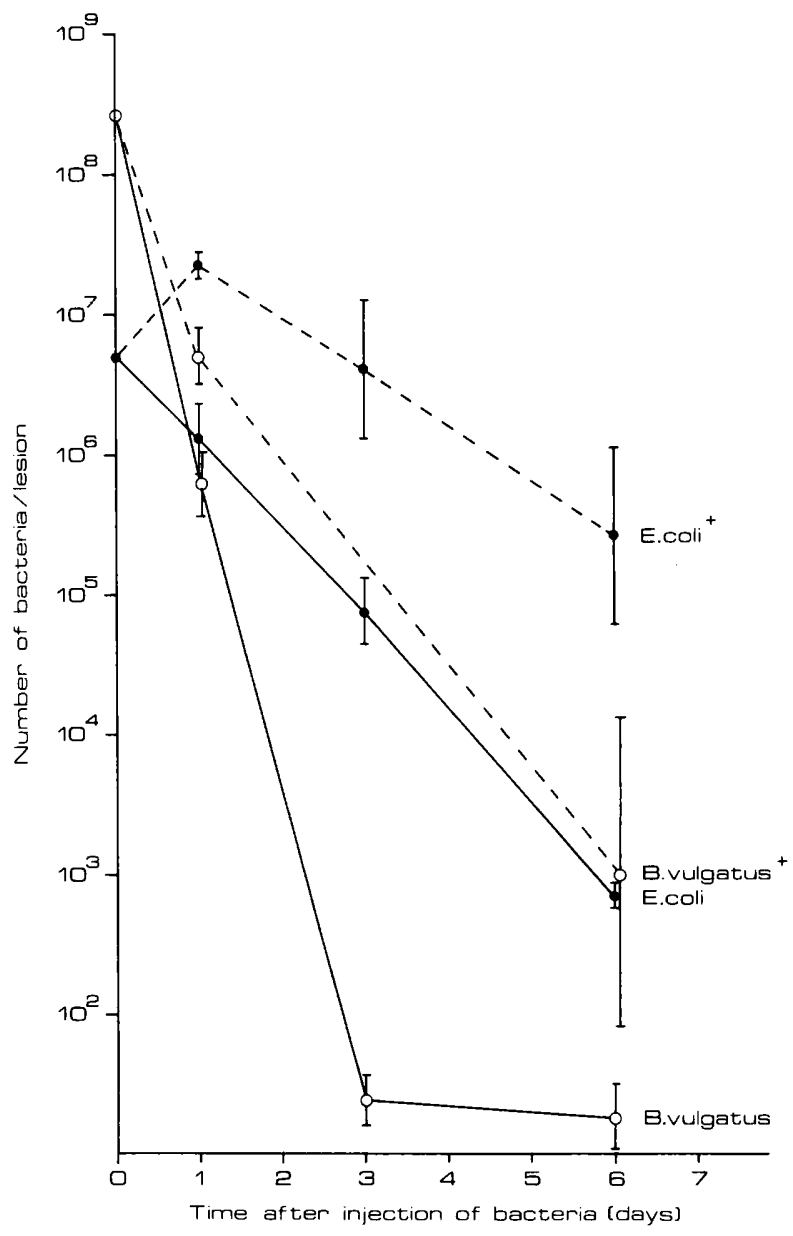

Fig. 3. - The clearance of E. coli strain EB $1(\bullet)$ and B. vulgatus strain BE $18(O)$ after injection alone $(-)$ or together $(---)$. Each point represents the mean of at least six lesions and bars indicate the SEM. 


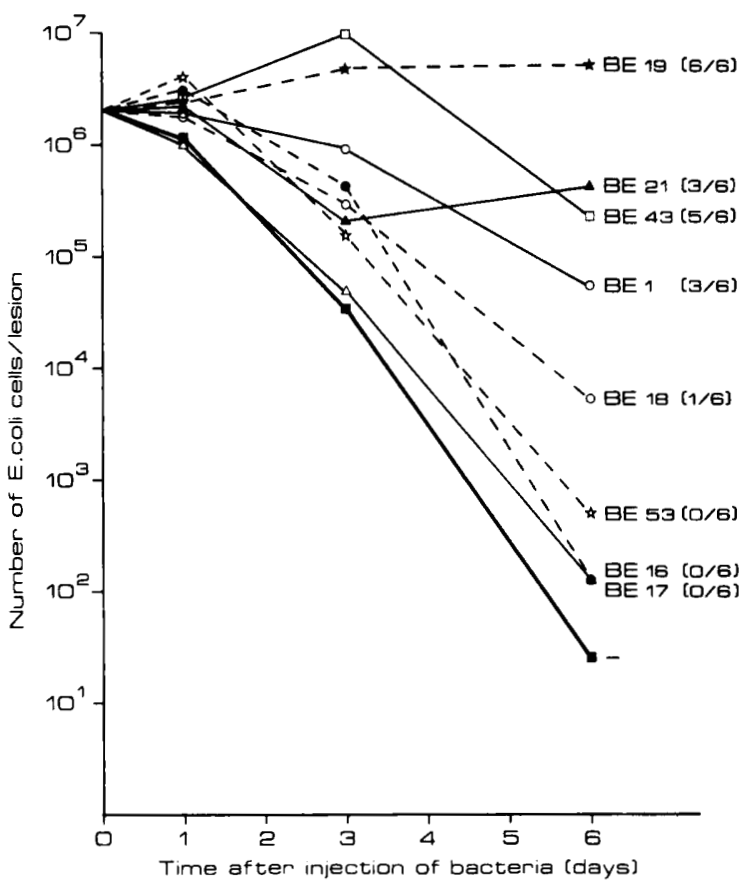

Fig. 4. - The influence of four strains of $B$. fragilis (-) and $B$. vulgatus (-- ) on the clearance of $E$. coli (control $E$. coli alone - - ). Each point represents the mean of at least six lesions. The numbers of abscesses at day 6 are given in parentheses.

marked with $B$. fragilis strains BE $1, \mathrm{BE} 21$ and $\mathrm{BE}$ 43 and $B$. vulgatus strain BE 19. The clearance of Bacteroides strains in mixed infections with $E$. coli was also impaired in these four combinations. The data with regard to the numbers of Bacteroides strains at day 6 are shown (fig. 5). In the other combinations where Bacteroides strains influenced but little the clearance of $E$. coli, their own clearance was enhanced by the presence of $E$. coli.

That the observed pathogenic synergy was not specific for Bacteroides species was seen in tests with $S$. aureus together with $E$. coli. Although the clearance of $S$. aureus strain BE 50 was only slightly influenced by the presence of $E$. coli, the effect of $S$. aureus on the clearance of $E$. coli was comparable to that of B. fragilis (data not shown).

\section{Discussion}

The higher incidence of $B$. fragilis in combination with $E$. coli in mixed infections, despite its lower prevalence in the normal intestinal flora may be explained either by the higher virulence of this species or by invoking some mechanism involving pathogenic synergy specific for $B$. fragilis.

The virulence of Bacteroides strains was readily

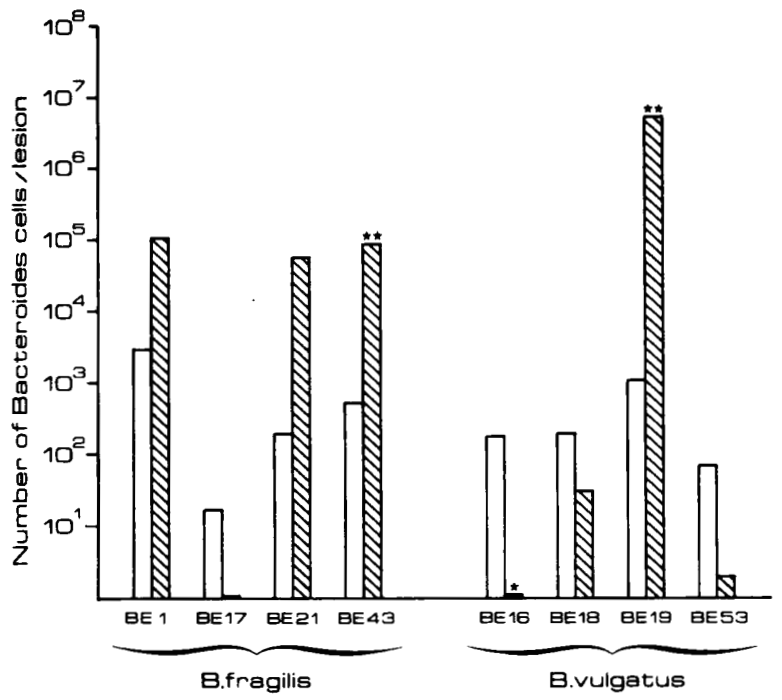

Fig. 5. - The numbers of $B$. fragilis and B. vulgatus in the skin of mice 6 days after injection alone $(\square)$ or together with $E$. coli strain EB 1 (2). The significance is indicated as ${ }^{* *}$ at a level of $p=0.01$ and as * at a level of $\mathrm{p}=0.05$.

measured by following their clearance from the subcutaneous tissue of mice as long as logarithmicphase bacteria were used. Lack of reproducibility in experiments with stationary-phase cultures of $\mathrm{Bac}$ teroides was attributable to the presence of variable numbers of non-viable cells in stationary-phase cultures. The finding that $B$. fragilis was generally more virulent than $B$. vulgatus is in full agreement with that of Maskell (1981) who also used a mouseskin model. It has been proposed that the capsular polysaccharide of $B$. fragilis is an important virulence factor (Onderdonk et al., 1977). However, the presence of a capsule is not a unique character of $B$. fragilis strains. Other authors have reported that other members of the fragilis group produce capsules (Babb and Cummins, 1978) which also may be important virulence factors (Brook and Walker, 1984). The strains of B. fragilis and B. vulgatus used in this study also showed varying degrees of capsulation when observed in India-ink preparations and it was surprising that correlation between degrees of encapsulation and virulence for mice was not observed in our experiments, a situation that clearly requires further study.

Four strains each of $B$. fragilis and B. vulgatus, tested for their infectivity for mice in combination with $E$. coli, tended to show an 'all-or-nothing' effect. At certain doses, abscesses developed containing large numbers of both species; at other doses the lesions, containing relatively small numbers of bacteria, tended to heal. This observation suggests 
that a critical dose of both species must be maintained for at least a certain period for successful abscess formation. Depending on dose, both $B$. fragilis and $B$. vulgatus caused abscess formation along with $E$. coli whereas, injected alone, they did not. The influence of Bacteroides strains other than strain BE 19 merely reflected their own virulence. From these findings we conclude that the influence of Bacteroides strains on the clearance of $E$. coli is not specific for $B$. fragilis but rather is a non-specific phenomenon dependent on the ability of the anaerobe to maintain itself for at least a few days and results with $S$. aureus confirmed this idea. The clearance of Bacteroides strains in mixed infections is reduced in infections resulting in abscess formation. The presence of $E$. coli obviously created a niche favourable for Bacteroides spp. In other circumstances the presence of $E$. coli in mixed infections is rather disadvantageous for Bacteroides spp.

One mechanism of pathogenic synergy between E. coli and B. fragilis that has been studied in vitro is the inhibition of phagocytosis of $E$. coli in the

\section{REFERENCES}

Babb J L, Cummins C S 1978 Encapsulation of Bacteroides species. Infection and Immunity 19:1088-1091.

Brook I, Walker R I 1984 Significance of encapsulated Bacteroides melaninogenicus and Bacteroides fragilis groups in mixed infections. Infection and Immunity 44:12-15.

Finegold S M 1977 Anaerobic bacteria in human disease. Academic Press, New York, p 257.

Gorbach S L, Bartlett J G 1974a Anaerobic infections, part 1. New England Journal of Medicine 290:1177-1184.

Gorbach S L, Bartlett J G $1974 b$ Anaerobic infections, part 2. New England Journal of Medicine 290:1289-1294.

Kelly M J 1978 The quantitative and histological demonstration of pathogenic synergy between Escherichia coli and Bacteroides fragilis in guinea pig wounds. Journal of Medical Microbiology 11:513-523.

MacLaren D M, Namavar F, Verweij-van Vught A M J J, Vel W A C, Kaan J A, 1984 Pathogenic synergy: mixed intraabdominal infections. Antonie van Leeuwenhoek 50:775787.

Maskell J P 1981 The pathogenicity of Bacteroides fragilis and related species estimated by intra-cutaneous infection in the guinea-pig. Journal of Medical Microbiology 14:131-140.

Namavar F, Verweij-van Vught A M J J, Bal M, van Steenbergen T J M, de Graaff, J, Maclaren D M 1983 Effect of anaerobic presence of the anaerobe which competes for complement factors (Tofte et al., 1980; Namavar et al., 1983; Vel et al., 1985). Mouse serum also showed a reduced capacity for opsonisation of $E$. coli and Zymosan after incubation with $B$. fragilis strain $\mathrm{BE}$ 1 or B. vulgatus strain BE 18; in this respect, differences were not observed between these strains (data not shown). These observations may mean that both $B$. fragilis and $B$. vulgatus can act synergistically with $E$. coli in mice by competing for complement but that other factors determine their virulence and, therefore, the actual number of cells and the length of time they are available in the tissues for complement consumption. Thus, $B$. vulgatus may be unable to express in-vivo synergy with $E$. coli. It still remains to be proven, however, that complement depletion observed in vitro also occurs in vivo. The factors responsible for the higher virulence of $B$. fragilis strains are currently under investigation.

The skilful technical assistance of $\mathrm{Mr} \mathrm{W}$. Schouten was greatly appreciated.

bacteria on killing of Proteus mirabilis by human polymorphonuclear leukocytes. Infection and Immunity 40:930-935.

Onderdonk A B, Bartlett J G, Louie T, Sullivan-Seigler N, Gorbach S L 1976 Microbial synergy in experimental intraabdominal abscess. Infection and Immunity 13:22-26.

Onderdonk A B, Kasper D L, Cisneros R L, Bartlett J G 1977 The capsular polysaccharide of Bacteroides fragilis as a virulence factor: Comparison of the pathogenic potential of encapsulated and unencapsulated strains. Journal of Infectious Diseases 136:82-89.

Shah H N, Williams R A O, Bowden G H, Hardy J M 1976 Comparison of the biochemical properties of Bacteroides melaninogenicus from human dental plaque and other sites. Journal of Applied Bacteriology 41:473-492.

Tofte R W, Peterson P K, Schmeling D, Bracke J, Kim Y, Quie P G 1980 Opsonization of four Bacteroides species: role of the classical complement pathway and immunoglobulin. Infection and Immunity 27:784-792.

Vel W A C, Namavar F, Verweij-van Vught A M J J, Pubben A N B, MacLaren D M 1985 Killing of Escherichia coli by human polymorphonuclear leukocytes in the presence of Bacteroides fragilis. Journal of Clinical Pathology 38:86-91. Verweij-van Vught A M J J, Namavar F, Sparrius M, Vel W A C, MacLaren D M 1985 Pathogenic synergy between Escherichia coli and Bacteroides fragilis: studies in an experimental mouse model. Journal of Medical Microbiology 19:325-331. 